\title{
Anotaciones de la ecología reproductiva del Gunguma Pimelodus sp. en la Ciénaga El Tigre, cuenca media del rio Atrato Chocó, Colombia
}

\section{Goal of reproductive ecology Pimelodus sp. «Gunguma» in the Swamp El Tigre, Atrato River middle basin Chocó, Colombia}

\section{Eric Yair Cuesta-Ríos ${ }^{1}$, Marleny Cuesta Incel ${ }^{2}$}

\section{Resumen}

Se estudian y analizan aspectos reproductivos de Pimelodus sp «Gunguma» en el complejo cenagoso El Tigre (cuenca media del río Atrato, Colombia), durante seis meses comprendidos entre enero y junio del año 2010. Se capturaron 166 individuos, donde la proporción mostró una mayor cantidad de hembras, datos que expusieron proporciones estadísticamente significativas; los intervalos de peso fluctuaron entre 256 y $612 \mathrm{~g}$ y los de longitud estándar de 10,3 a $32.5 \mathrm{~cm}$. Los peces en su mayoría se capturaron maduros (III), estimando su talla de madurez media en $24 \mathrm{~cm}$, para sexos combinados; al no tomar el ciclo reproductivo completo de la especie se proyectó que su pico de madurez sexual está entre mayo y junio y que posiblemente el desove se presente entre julio y agosto, época donde se presentaron los más altos valores de precipitación para el 2010. La relación gonadosomática fluctuó mensualmente para las hembras machos y sexos combinados. Para futuras investigaciones con el morfo se recomienda un análisis taxonómico de la especie, en búsqueda de analizar con más detalle aspectos de la conducta reproductiva y trófica de Pimelodus sp, además de realizar prospecciones biológicas donde se correlacionen estos comportamientos con los factores ambientales, fisicoquímicos y antrópicos en la cuenca hidrográfica del río Atrato donde se haya registrado la presencia de la especie.

Palabras clave: Ciénaga El Tigre; Estado de madurez gonadal; Gumguma; Índice gonadosomático; Pimelodus sp.

\begin{abstract}
We studied and analyzed reproductive aspects of Pimelodus sp «Gunguma» swamp complex in El Tigre, (middle basin of the Atrato River, Colombia), for six months between January and June 2010. 166 individuals were captured, wherein the ratio showed a greater number of females who presented data statistically significant proportions. Intervals weights ranged between 256 and $612 \mathrm{~g}$ and standard length of 10.3 to $32.5 \mathrm{~cm}$. The fish were caught mostly mature (III), estimating their size at maturity in $24 \mathrm{~cm}$, for sexes combined, by failing to complete the reproductive cycle of the species is projected to peak at sexual maturity is between May and June and possibly spawning occurs between July and August, time where we presented the highest values of precipitation for 2010. The gonadal somatic fluctuated monthly for males and females combined sexes. For future research it is recommended to morph taxonomic analysis of the species, seeking further analyze aspects of reproductive behavior and trophic Pimelodus sp, bioprospecting in addition to where these behaviors are correlated with environmental factors, physicochemical, and
\end{abstract}

1 Instituto de Investigaciones Ambientales del Pacifico (IIAP). Grupo Investigación Conocimiento, Manejo y Conservación de los Ecosistemas del Chocó Biogeográfico, Quibdó, Colombia. e-mail: cuestarios@gmail.com

2 Bióloga con Énfasis en Recursos Naturales. Universidad Tecnológica del Chocó, Quibdó, Chocó, Colombia. e-mail: marcuin84@hotmail.com Recibido: 7 de febrero de 2012 Aceptado: 3 de abril de 2012 
man in the Atrato River basin which have recorded the presence of the species.

Keywords: Swamp El Tigre; Gonadal maturity stage; Gumguma; Gonadosomatic index; Pimelodus sp.

\section{Introducción}

Las aguas continentales de Colombia son poseedoras de una gran variedad de especies ícticas de gran importancia económica y comercial que están siendo sometidas a una fuerte presión pesquera, que en algunos casos ha llevado a una disminución notable de sus poblaciones, desconociéndose de muchas especies la biología y ecología básica de su medio, lo que impide el aprovechamiento racional de este importante recurso (Arias 1996). Entre los peces del género Pimelodus, conocidos comúnmente como «barbudos, encontramos al Gunguma (Pimelodus sp) que es una especie que presenta importancia económica, siendo de interés especial en la pesca de subsistencia, aunque ocasionalmente llega a los mercados en cantidades considerables, representando en las pesquerías del Atrato un importante renglón, pero no tan significativo como otros bagres reofilicos (bagre sapo, charre, barbudo y nicuro) (Negrete y Santos 2005; CCI e INCODER 2006).

El Gunguma Pimelodus sp, que representa una importante fuente abastecedora de proteína animal para los pobladores de las riberas del río Atrato y sus afluentes, debido quizás a la palatabilidad de su carne, convirtiéndolo en una especie promisoria con altos potenciales en el aprovechamiento piscícola. Este comportamiento de consumo hace que la pesca sea un soporte importante de seguridad alimentaria para las comunidades ribereñas del río Atrato, requiriéndose primordialmente para su aprovechamiento el conocimiento bioecológico de las especies, como propósito de amortiguar la sobrexplotación y asegurar el bienestar en su medio, buscando integralmente que los recursos tengan vigencia biológica y sigan cumpliendo su papel como soporte del desarrollo económico.

Son escasos o nulos los estudios sobre la biología del Gunguma en el río Atrato, porque aún no se ha determinado la especie, lo que requiere de una completa revisión sistemática del género. Información sobre el Gunguma se encuentra en trabajos aislados de inventarios ícticos, separados del conocimiento bioecológico de la especie. Así que este trabajo se convierte en el primer paso para el conocimiento de la misma y de esta manera proyectar futuras investigaciones en taxonomía, bioecología y aspectos de pesca. Lo anterior pone de manifiesto que se requiere de estudios que aporten al conocimiento de la biología, ecología y pesca, información base para poder implementar medidas orientadas al aprovechamiento sostenible de la especie.

Siguiendo las apreciaciones anteriores se pretendió estudiar algunos aspectos biológicos y ecológicos del Gunguma Pimelodus sp, en la Ciénaga del Tigre, cuenca media del río Atrato, como elemento fundamental para el establecimiento de pautas de manejo del recurso íctico.

Área de estudio. El trabajo se desarrolló en la Ciénaga del Tigre ubicada en las inmediaciones del Corregimiento El Tigre, en el Municipio de Bojayá, a los 6 31' 25" N y los 76 58' 28"W, con una altura de 36 msnm (www.fallingrain.com). Pertenece a la zona de vida selva pluvial central. Ambientalmente corresponde a la zona de temperatura mega termal $\left(25,7^{\circ}\right.$ a $\left.27,9^{\circ} \mathrm{C}\right)$, zona de pluviosidad media (6.000 mm) y de balance hídrico moderadamente húmedo (25,0 a 112,0) (Poveda-M et al., 2004) (Figura 1).

La cuenca media del Atrato donde se está la Ciénaga El Tigre, exhibe una exuberante selva tropical, diversidad de flora y fauna así como en fuentes hídricas, enmarcadas en una serie de ciénagas que han representado para las comunidades aledañas a estas una fuente de sustento diario a través del tiempo, ofreciendo a sus habitantes diferentes actividades económicas como la pesca, agricultura y extracción de madera, predominando una economía de subsistencia,

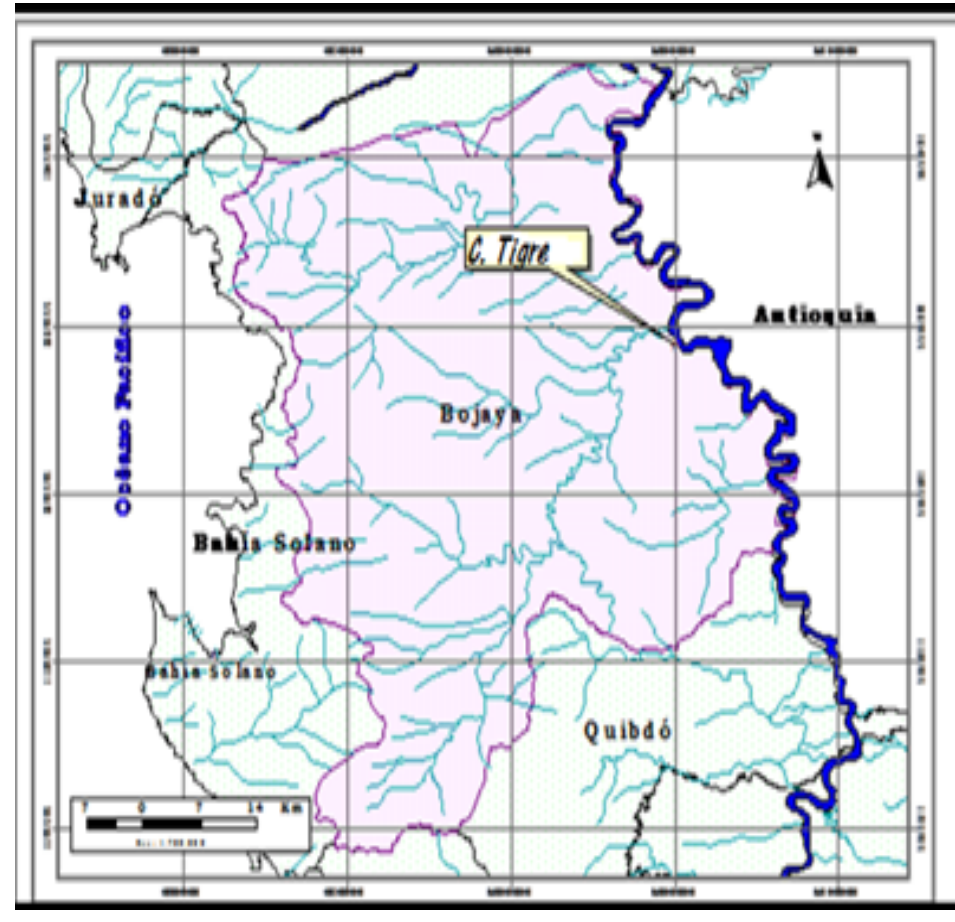

Figura 1. Ubicación geográfica de la Ciénaga El Tigre municipio de Bojayá. 


\section{Bioetnia Volumen 9 № 1 (enero-junio), 2012}

siendo la pesca la principal forma de garantizar proteína a las familias campesinas, convirtiendo a los peces en la mayor fuente vital de proteína e ingreso de dinero para las familias de las zonas rivereñas y de los que realizan actividades acuícolas (Marín 2002).

\section{Métodos}

Trabajo de campo y consideraciones para el levantamiento de la información. La fase de campo se desarrolló en el período comprendido entre enero y junio del año 2010; se tuvo en cuenta socializar el proyecto ante el consejo comunitario del corregimiento de El Tigre los cuales dieron sus apreciaciones sobre la problemática ambiental entorno al manejo y conservación del recurso íctico y procedieron a dar el visto bueno para poder realizar dicha actividad en su territorio el cual está bajo el título colectivo del Consejo Comunitario Mayor de la Asociación Campesina Integral del Atrato (COCOMACIA), todo esto con el fin de explicar los alcances y objetivos de la propuesta.

La colectas se llevaron a cabo, con una frecuencia de un muestreo mensual y una intensidad de ocho días, mediante la utilización de trasmallos, atarrayas, tolas y galandros, siendo este último el más efectivo para la captura de los ejemplares, a los cuales se les tomó registro de sus respectivas medidas como son longitud total (LT) y longitud estándar (LE) con una cinta métrica graduada en centímetros y el peso con una balanza con aproximación al gramo.

Aspectos reproductivos. A los ejemplares capturados para este estudio se les realizó un corte o incisión medio ventral, a partir de la abertura urogenital hasta los radios branquiostegales, de manera que se lograran extraer las gónadas para determinar el sexo y se conservaron en formol al 5\%, en recipientes plásticos para su posterior análisis en el laboratorio.

Desarrollo gonadal. Los estadíos de desarrollo gonadal se determinaron a través de la observación directa de características macroscópicas externas (presionando el poro urogenital de la hembra y la protuberancia carnosa del macho, «pene»), e internas mediante la extracción de las gónadas. Se determinó, de acuerdo con la escala de Nikolsky (1963) y modificada por Cala et al. (1996) como inmaduro (I), madurando (II), maduro (III) y post-desove (IV).

Tallas medias de madurez gonadal. Para determinar las tallas mínimas y medias de maduración sexual se tuvo en cuenta ejemplares machos y hembras en estadíos III y IV. El análisis de la talla media de madurez sexual se determinó sobre la base del criterio del 50\% de la fracción de individuos maduros (Arancibia et al., 1994), utilizando la siguiente ecuación logística (Echeverría, 1987): Pi = 1/1+ exp [r* (LE - lm)]. Donde Pi; es la proporción de individuos sexualmente maduros a la longitud estándar (LE), (r y lm) son constantes.
El ajuste de la función de madurez se realizó aplicando un modelo de regresión simple a través del programa estadístico SPSS versión 15.

Índice gonadosomático. El índice gonadosomático (IGS) se calculó mediante la ecuación: IGS = Pg/ Pp x 100, donde: $\mathrm{Pg}=$ peso de la gónada, $\mathrm{Pp}=$ peso total del pez descrita por Tresierra y Culquichicón (1995).

Fecundidad. Se obtuvo aplicando el método gravimétrico indirecto Laevastú (1971), mediante la ecuación F = Po x n/ $\mathrm{Pm}$, donde $\mathrm{Po}=$ peso de la masa ovárica, $\mathrm{n}=$ número de ovocitos maduros presentes en una porción de masa ovárica y $\mathrm{Pm}$ = peso de la porción de masa ovárica. Mediciones de ovocitos se realizaron con pie de rey electrónico marca Mitutuyo modelo No CD-6" C-D.

\section{Resultados y discusión}

Se colectaron 166 individuos con una biomasa total de $64.064 \mathrm{~g}$ y un intervalo entre 20 y $33 \mathrm{~cm}$ (LT) y 15 y $28 \mathrm{~cm}$ (LE).

Proporción de machos y hembras. En el transcurrir del estudio se pudieron evidenciar diferencias estadísticas significativas ( $\mathrm{p} \geq 0.05$ ), no siendo diferentes a la proporción esperada 2:1. Durante los meses de estadío fue predominante la proporción de las hembras sobre los machos, con excepción del mes de abril. No obstante los resultados logrados, machos 73 (43.5\%) ejemplares y hembras 93 (55.5\%) ejemplares, no permitió que se marcaran diferencias estadísticas significativas (Tabla 1 ).

La Tabla 1 exhibe la proporción sexual de Pimelodus sp durante los meses de estudio; las hembras presentan un

\section{Tabla 1}

\section{Proporción de machos y hembras de Pimelodus sp., enero-junio, 2010}

\begin{tabular}{crrr}
\hline Mes & Sexo & N & $\%$ \\
\hline \multirow{2}{*}{ Enero } & H & 22 & 13 \\
& M & 16 & 10 \\
Febrero & H & 20 & 12 \\
& M & 14 & 8 \\
Marzo & H & 27 & 16 \\
& M & 16 & 10 \\
Abril & $\mathrm{H}$ & 14 & 8 \\
& $\mathrm{M}$ & 21 & 13 \\
Mayo & $\mathrm{H}$ & 5 & 3 \\
& $\mathrm{M}$ & 3 & 2 \\
J unio & $\mathrm{H}$ & 5 & 3 \\
& $\mathrm{M}$ & 3 & 2 \\
Total & 166 & 100 & \\
\hline
\end{tabular}


dominio global sobre los macho, tanto en la época de lluvias bajas que comprendió de enero hasta marzo y la de lluvias ascendentes de abril que fue el mes con mayor proporción de machos hasta junio, período donde las proporciones de hembras disminuyeron notablemente hasta alcanzar una proporción cercana a 2:1. López-Casas y Jiménez-Segura (2007), en su estudio sobre reproducción y hábitos alimenticios del nicuro, $P$. blochii en la ciénaga de cachimbero, río Magdalena, Colombia, obtuvieron una proporción de 1:1, donde el análisis de la proporción sexual a la talla, indica que las hembras predominan a tallas mayores y los machos a tallas menores.

Estado y talla de madurez sexual e índice gonadosomático. La longitud en que el $50 \%$ de hembras y machos de Pimelodus sp alcanzaron la talla media de maduración, fue estimada en $24 \mathrm{~cm}$ (Figura 2). Estos datos son diferentes, a los obtenidos por López-Casas y Jiménez-Segura (2007), porque los individuos maduraron más tempranamente, donde las hembras maduras presentaron tallas entre los 13.2 y los $21.6 \mathrm{~cm}$ de LE y el único macho maduro capturado tenía $18 \mathrm{~cm}$ de LE.

Para co-genéricos, CCI y MADR 2007, determinan en la cuenca media del rio Magdalena, para Pimelodus grosskopfii, una TMM de 25,7 cm LE para las hembras ( $\mathrm{n}=688$ ), 23,7 cm para los machos $(\mathrm{n}=551)$ y $24,7 \mathrm{~cm}$ para los dos sexos combinados ( $\mathrm{n}=1239)$, indistintamente con Pimelodus clarias, se estableció la TMMG en 18,9 cm LS para las hembras $(\mathrm{n}=293), 16,7 \mathrm{~cm}$ para los machos ( $\mathrm{n}=192)$ y $17,7 \mathrm{~cm}$ para los dos sexos combinados $(\mathrm{n}=485)$.

La presencia de individuos en maduración y maduros en todos los meses de estudio, permite inferir que quizás entre enero y junio el gunguma está en su ciclo reproductivo, de julio a septiembre su desove y de octubre a diciembre se encuentran inmaduros o en recuperación. Carolsfeld et al. (2004), Galvis et al. (1997), y Mojica y Jiménez, (2001), señalan que las poblaciones de este género en otras cuencas con régimen hidrológico monomodal, presentan una estrategia reproductiva estacional, con un solo desove a lo largo de todo el año, asociado con movimientos migratorios entre los ríos y su plano inundable en busca de los lugares de desove. Esta afirmación posiblemente este ratificando los resultados de este estudio cuando se aduce sobre la época de maduración de la especie que solo se estudió en seis meses del año y que si quizás se hubiese cubierto los 12 meses del año se tendrían datos más precisos de su conducta reproductiva.

Analizando estos datos desde un supuesto, tendríamos a partir del aumento progresivo de la maduración, que de enero a marzo, comienzo del ciclo reproductivo, de abril a junio, gónadas maduras, entre julio y agosto desove, en septiembre están en reposo y de octubre a diciembre están en un estado de inmadurez. Datos ratificados por Rodríguez y Mojica (2005), quien señala que el pico de maduración gonadal del co-genérico gunguma ( $P$. blochii) está comprendido entre mayo y junio, pero estos resultados van levemente contrariados con los de López-Casas y Jiménez-Segura (2007) que determinaron que durante marzo y mayo, la población de $(P$. blochii) estuvo representada por un mayor número de hembras en estado B (en maduración) y machos en estado E (en reposo). En octubre, la mayoría de hembras capturadas se encontraron en estado $\mathrm{C}$ (maduras, próximas a desovar) y los machos se encontraron en su mayoría en estado B.

Al confrontar los análisis expuestos antes con los resultados obtenidos en la presente investigación, se encuentran similitudes y diferencias, estas últimas pueden estar marcadas por la influencia del fenómeno de la niña durante el año 2010, que quizás alteró el ciclo reproductivo de la especie por sus constantes e intempestivas fluctuaciones hidroclimáticas e hidrológicas.

Al analizar el índice gonadosomático (IGS) se encontró

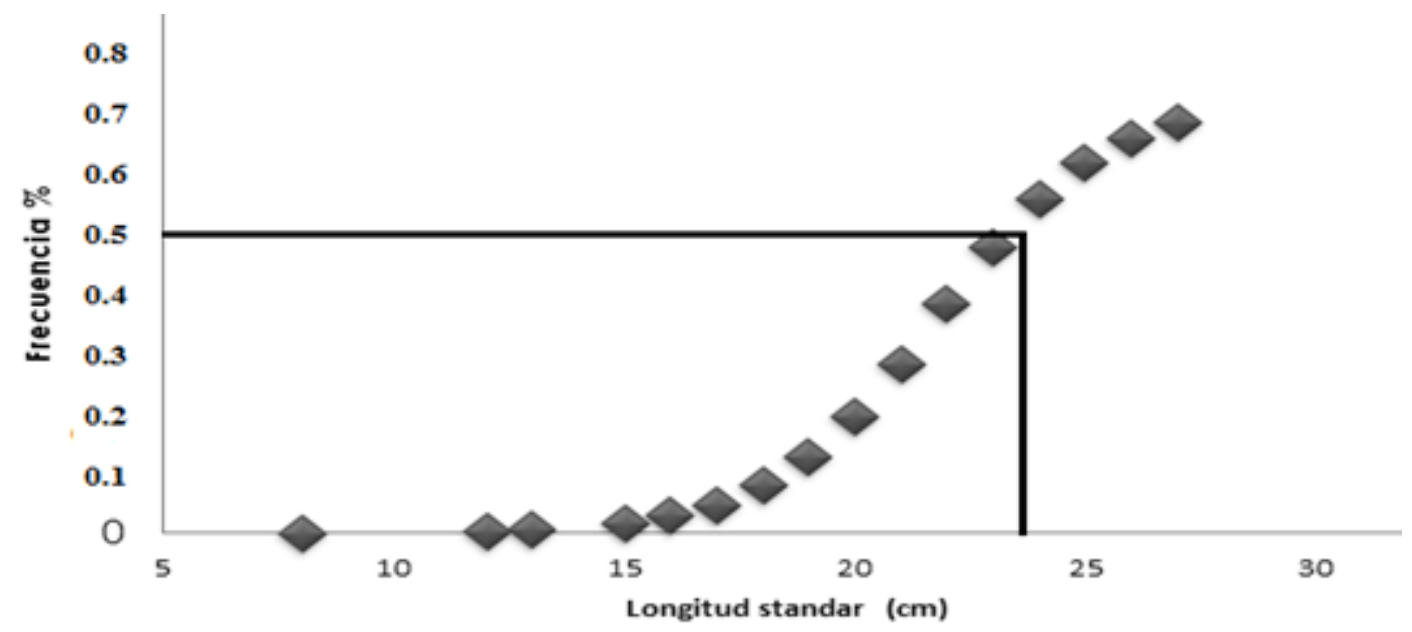

Figura 2. Talla de maduración media del Pimelodus sp. en la Ciénaga El Tigre, cuenca media río Atrato. 
que este es mayor en las hembras; los mayores valores se presentaron en los meses mayo y junio para ambos sexos (Figura 3), siendo este su pico de madurez, a diferencia lo reportado por López-Casas y Jiménez-Segura (2007), río Magdalena, quienes señalan el pico de madurez gonadal de $P$. blochii, entre marzo y mayo.

Rodríguez y Mojica (2005), manifiestan que el ciclo reproductivo de algunos Pimelodus, se encuentra influido por factores ambientales. La maduración gonadal empieza en la subida de aguas y alcanza la fase máxima de maduración entre mayo y junio, correspondiente a los caudales máximos registrados, tal como lo indica el índice gonadosomático. Castro (1986) señala que la mayoría de bagres efectúan migraciones reproductivas conocidas como subienda, las cuales empiezan cuando los niveles de los ríos han disminuido y el pez va buscando las áreas de dispersión, en donde finaliza su maduración gonadal; luego se dirigen al cauce principal en donde realizan el desove masivo asociado con las lluvias y altos niveles del caudal de los ríos.

En relación con la fecundidad y diámetro de los ovocitos de Pimelodus sp, los conteos arrojaron un promedio de 15.885 ovocitos por hembra, presentando un rango de 0.48 a $0.6 \mathrm{~mm}$ de diámetro; estos resultados son similares a los reportados por Rodríguez (2003) quien determinó que el diámetro de los ovocitos maduros está comprendido entre 480 y $600 \mu$.

La fecundidad relativa fue de $662 \pm 78$ ovocitos g, igualmente estos resultados no están muy lejanos al promedio de ovocitos reportados por Beltrán-Ortega y Olaya-Nieto 2005) con un promedio de ovocitos de 15.264 ovocitos por hembra para $P$. clarias en el rio Sinú.

\section{Conclusiones}

En las poblaciones de Pimelodus sp, los sexos se encuentran proporcionalmente distribuidos, registrando diferencias significativas en la población de 73 (43.5\%) machos y 93 (55.5\%) hembras con una proporción de 2:1.

La talla media de madurez sexual para Pimelodus sp, fue determinada en $24 \mathrm{~cm}$ de longitud estándar, para hembras y machos respectivamente, asociando su comportamiento reproductivo al ciclo hidrológico del río Atrato, coincidiendo la maduración sexual con la época de lluvias bajas a lluvias ascendentes de enero hasta abril y posiblemente el desove con la época de creciente de julio y agosto.

\section{Recomendaciones}

Ante todo lo primero que se requiere es una análisis taxonómico de la especies, lo cual lleve a la realización de estudios de la conducta reproductiva de Pimelodus sp, que cubran el ciclo reproductivo completo de la especie en diferentes épocas del año y otros ecosistemas acuáticos de tipo lentico o lotico, para poder establecer la incidencia de otros factores sobre la fisiología y bioecología de la especie, además de la ejecución de estudios de la correlación e incidencia de los factores ambientales, fisicoquímicos y antrópicos sobre la conducta y biología reproductiva de Pimelodus sp en la cuenca hidrográfica del río Atrato donde se haya registrado la presencia de la especie.

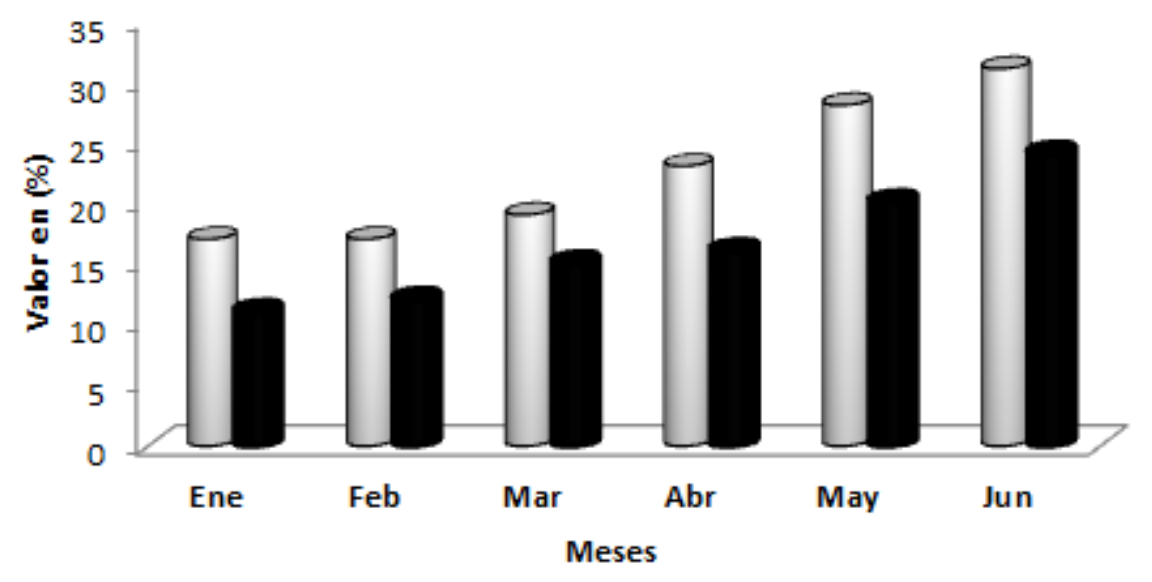

$\square$ Hembras Machos

Figura 3. Porcentajes del I GS por meses del Pimelodus sp. en la Ciénaga El Tigre cuenca media del río Atrato. 


\section{Ecología reproductiva del Pimelodus sp. EY. Cuesta-Ríos, M Cuesta Incel}

\section{Literatura citada}

Arias. J. A. 1996. Los peces de los Llanos. Anatomía, histología, y fisiología de la Sapuara, Semaprohilodus laticep c.f. Dahlia. Rev Asoc Colomb Ictiol. 1: 15-21.

Beltrán-Ortega, E. 2005. Biología reproductiva del Barbul (Pimelodus clarias f.c. Bloch 1785) en el río Sinú, Colombia. Trabajo de pregrado. Lorica: Universidad de Córdoba. 32 pp.

Cala, P., C. Pérez, J. Rodríguez. 1996. Aspectos biológicos de la población del Capaz, Pimelodus grosskopfii (Pises: Pimelodidae) en el embalsa de Betania y parte alta del río Magdalena, Colombia. Rev Acad Colomb Cienc. 20 (77): 319-30.

Carolsfeld, J., B. Harvey, C. Ross, A. Baer (eds.). 2004. Migratory fishes of South America: biology, fisheries and conservation status. Washington, Ottawa: World Fisheries Trust/World Bank/IDRC. 372 pp.

Castro, D. M. 1986. Los bagres de la subfamilia Sorubiminae de la Orinoquia y Amazonia colombiana. (Siluriformes, Pimelodidae). Bol Ecotropica. 13: $1-40$.

Corporación Colombia Internacional (CCI) e Instituto Colombiano de Desarrollo Rural (INCODER). 2006. Informe de pesca y acuicultura. Bogotá: CCI, INCODER. 136 p.

Galvis, G., I. Mojica, M. Camargo. 1997. Peces del Catatumbo. Bogotá: Asociación Cravo Norte. 72p. Disponible en: http://www.fallingrain.com/ world/CO/11/El_Tigre.html

Laevastú, T. 1971. Manual de métodos de biología pesquera. Zaragoza: Editorial Acribia. 243 p.
López-Casas, S., L. F. Jiménez-Segura. 2007. Reproducción y hábitos alimenticios del nicuro, Pimelodus blochii (Valenciennes, 1840) (Pisces: Pimelodidae), en la ciénaga de Cachimbero, río Magdalena, Colombia. Actual Biol. 29 (87): 193-201.

MADR, CCI. 2007. Pesca y acuicultura. Colombia 2006. Bogotá: Convenio Corporación Colombia Internacional, Ministerio de Agricultura y Desarrollo Rural.

Marín, J. 2002. Mujeres en la pesca artesanal. Comunidades negras del Bajo Atrato, Departamento del Chocó, Colombia. Rev Semillas. 16/17: 22-4.

Negrete, C., L. Santos. 2005. Acción desde el INCODER en la cuenca media del río Atrato y el manejo de la estadística pesquera. Rivas, T., C, Rincón, H. Mosquera (Eds.). Memorias del VIII simposio colombiano de ictiología. Quibdó: Edit. Servicios Gráficos Integrales Colombia.

Nikolsky, G.V. 1963. The ecology of fishes. London, New York: Academic Press. 352 pp.

Poveda, C, C. Rojas, A. Rudas, O. Rangel-Ch. 2004. Climas del Chocó Biogeográfico de Colombia. pp 39-89. En Rangel, J. (Ed). Colombia Diversidad Biótica IV. Chocó Biogeográfico/Costa Pacífica. Bogotá: Universidad Nacional de Colombia. Editorial Universidad Nacional de Colombia. 997 pp.

Rodríguez, J. A., H. O. Mojica. 2005. Preproducción y manejo de silúridos en cautiverio. En: Daza, P.V., M. Á, Landines, A. I. Sanabria (Eds.) Reproducción de los peces en el trópico. Bogotá: INCODER-Universidad Nacional de Colombia. 241pp.

Tresierra, A., Z. Culquichicón. 1995. Manual de biología pesquera. Trujillo: Concytec. p. 62-5. 